\title{
Üçüncü mertebeden gecikmeli bir diferansiyel denklem için çözümlerin sürdürülebilirliği ve sinırlılığ1
}

\author{
Timur AYHAN* \\ Adiyaman Simya Koleji, Adiyaman \\ Geliş Tarihi (Received Date): 14.05 .2020 \\ Kabul Tarihi (Accepted Date): 11.12.2020
}

$\ddot{O} z$

Bu çalışmada Lyapunov'un ikinci metodu kullanılarak üçüncü mertebeden gecikmeli bir diferansiyel denklemin çözümlerinin sinırlılı̆̆l ve sürdürebilirliği ile ilgili yeni sonuçlar elde edilmiştir. Bu çalışma literatürde üçüncü mertebeden gecikmeli ve gecikmesiz diferansiyel denklemler üzerine iyi bilinen bazı sonuçları kapsamış ve daha ileri götürmüş̧ür. Ayrıca çalışmada elde ettiğimiz sonuçların daha iyi anlaşılması için bir örnek verilmiştir.

Anahtar kelimeler: Lyapunov fonksiyonu, sürdürebilirlik, sinırlılık, üçüncü mertebe.

\section{Continuability and boundedness of solutions to a differential equation of third order with multiple deviating arguments}

\begin{abstract}
In this study, Lyapunov's second method is used to obtain criteria for continuability and boundedness of solutions to a kind of third order differential equations with multiple deviating arguments. The result obtained in this work includes and extends some well known results on third order differential equations with and without delay in the literature. We also give an example for better understanding our result.
\end{abstract}

Keyvords: Lyapunov function, continuability, boundedness, third order.

\footnotetext{
*Timur AYHAN, tayhan002@gmail.com, http://orcid.org/0000-0002-3105-7806
} 


\section{Giriş}

Belirtmek gerekir ki üçüncü mertebeden gecikmeli diferansiyel denklemler bilim ve teknolojinin çeşitli alanlarındaki problemlerinin matematiksel modellenmesinde büyük bir öneme sahiptir. $\mathrm{Bu}$ yüzden üçüncü mertebeden lineer olmayan gecikmeli diferansiyel denklemlerin çözümlerinin niteliksel davranışları birçok araştırmacı tarafından incelenmiş ve literatürde bu konuyu tartışmak için birçok farklı metot geliştirilmiştir. $\mathrm{Bu}$ kapsamda en önemli metotlardan biri Lyapunov [1] tarafindan geliştirilen Lyapunov' un ikinci metodu olarak bilinir. Bu çalışmada, adi diferansiyel denklemlerin çözümleri hakkında herhangi bir bilgi olmaksızın Lyapunov' un ikinci metodu direkt olarak uygulanabildiğinden direkt metot olarak da bilinen bu metot kullanılmıştır. Genel bir yöntem olmadığından dolayı gecikmeli diferansiyel denklemler için Laypunov fonksiyonelini oluşturmak oldukça zordur. Günümüzde, bu metot gecikmeli ve gecikmesiz diferansiyel denklemlerin çözümlerinin kararlılığı, kararsızlığı, sınırlılığı, sürdürebilirliği, periyodikliği ve yakınsaklığı gibi birçok farklı davranışının araştırılmasında çok önemli bir araç olarak dikkat çekmektedir. Gecikmeli diferansiyel denklemlerin çözümlerinin niteliksel davranışları üzerine yapılmış seçkin çalışmalar için Ahmad and Rama Mohana Rao [2], Burton [3], Driver [4], Hale [5], Krasovskii [6], Reissig vd. [7] ve Yoshizawa [8] tarafından hazırlanmış kitaplar incelenebilir. Ayrıca konunun önemi üzerine genel sonuçları içeren Abou-El-Ela vd. [9], Ademola vd. [10], Ademola [11], Ayhan [12], Ayhan ve Sofuoglu [13], Remili ve Oudjedi [14-15], Remili ve Beldjerd [16], Tunç ve Ayhan [17-20], Tunç [21] ve bu çalışmada atıfta bulunulan diğer çalışmalar incelenebilir. Bu çerçevede son zamanlarda Remili ve Beldjerd [16] tarafından $r(t)>0$ olmak üzere

$$
\begin{aligned}
& \left(\varphi(x) x^{\prime}\right)^{\prime \prime}+a(t) \psi\left(x^{\prime}\right) x^{\prime \prime}+b(t) f\left(x^{\prime}\right)+c(t) h(x(t-r(t)) \\
& =e\left(t, x(t), x\left(t-r(t), x^{\prime}\left(t-r(t), x^{\prime \prime}(t)\right)\right.\right.
\end{aligned}
$$

üçüncü mertebeden Lineer olmayan değişken gecikmeli diferansiyel denklemini incelenmiş ve bu denklemin tüm çözümlerinin düzgün asimptotik kararlılığını ve sınırlılığını garanti edecek bazı yeter şartlar elde edilmiştir. Bu çalışmada (1) denkleminin yerine $\eta_{i}(t)>0$ olmak üzere,

$$
\left(\varphi(x) x^{\prime}\right)^{\prime \prime}+a(t) f\left(t, x, x^{\prime}\right) x^{\prime \prime}+b(t) g\left(x, x^{\prime}\right)+c(t) \sum_{i=1}^{n} h_{i}\left(x\left(t-\eta_{i}(t)\right)\right)=p(t)
$$

biçiminde tanımlanan üçüncü mertebeden lineer olmayan değişken gecikmeli diferansiyel denkleminin çözümlerinin sınırlılığı ve sürdürülebilirliği gösterilecektir. (2) denklemi,

$$
\begin{aligned}
& x^{\prime}=\frac{y}{\varphi(x)} \\
& y^{\prime}=z, \\
& z^{\prime}=p(t)-\frac{a(t)}{\varphi(x)} f\left(t, x, \frac{y}{\varphi(x)}\right) z+\frac{a(t) \varphi^{\prime}(x)}{\varphi^{3}(x)} f\left(t, x, \frac{y}{\varphi(x)}\right) y^{2}-b(t) g\left(x, \frac{y}{\varphi(x)}\right) \\
& -c(t) \sum_{i=1}^{n} h_{i}(x(t))+c(t) \sum_{i=1}^{n} \int_{t-\eta_{i}(t)}^{t} \frac{y(s)}{\varphi(x(s))} h_{i}{ }^{\prime}(x(s)) d s
\end{aligned}
$$


şeklinde bir sistem olarak ifade edilsin. Burada $t \in \mathbb{R}^{+}, \mathbb{R}^{+}=[0, \infty)$ olmak üzere $\eta_{i}$ sürekli, türevlenebilir ve sinırlı gecikme değişkenleri, $\xi$ ise daha sonra tanımlanacak pozitif bir sabit olup $0 \leq \eta_{i}(t) \leq \xi, \eta_{i}{ }^{\prime}(t) \leq \rho,(0<\rho<1)$ dir. Ayrica $a, b, c, p \in$ $C^{1}\left(\mathbb{R}^{+},[0, \infty)\right), \varphi \in C^{1}(\mathbb{R},(0, \infty)), f \in C\left(\mathbb{R}^{+} \times \mathbb{R} \times \mathbb{R}, \mathbb{R}^{+}\right), g \in C\left(\mathbb{R} \times \mathbb{R}, \mathbb{R}^{+}\right)$ve $h \in$ $C^{1}(\mathbb{R}, \mathbb{R})$ dir.

Literatürde, lineer olmayan yüksek mertebeli gecikmeli ve gecikmesiz diferansiyel denklemlerin çözümlerinin niteliksel davranışları üzerine birçok çalışma olmasına rağmen Remili ve Beldjerd [16] tarafından incelenen (1) denkleminden ilham alınarak elde edilen (2) denkleminin çözümlerinin sınırlılığı ve sürdürülebilirliği şimdiye kadar hiçbir araştırmacı tarafından incelenmedi. $\mathrm{Bu}$ çalışma ile bu anlamda literatüre bir yenilik katmış olacağız. Remili ve Beldjerd [16] tarafından (1) denklemine uygun yeni bir Lyapunov fonksiyoneli elde edilerek Lypunov'un ikinci metodu yardımıyla bu denklemin çözümlerinin sınırlılığı ve kararlılığı ile ilgili yeni sonuçlar ortaya koyuldu. $\mathrm{Bu}$ çalışmada şimdiye kadar ele alınmamış ve (1) denkleminden daha genel (2) denklemi göz önüne alındı. Ayrıca bu denkleme uygun, orijinal bir Lyapunov fonksiyoneli inşa edilerek Lyapunov'un ikinci metodu yardımıyla denklemin çözümlerinin sınırlılığıyla birlikte Remili ve Beldjerd [16] tarafından incelenmeyen çözümlerin sürdürülebilirliği üzerine yeni bir sonuç elde edildi. Bununla birlikte ele alınan (2) denklemi, Tunç [21] tarafından incelenen denklemden daha genel ve Remili ve Oudjedi [15] tarafindan incelenen denklemden tamamen farklı olup bu denklemin çözümlerinin sürdürülebilirliği ile ilgili elde edilen sonuç, Tunç [21], Remili ve Oudjedi [15] ve bu alanda çalışan birçok araştırmacıya ele aldıkları denklemlerin çözümlerinin farklı bir niteliksel davranışı olan çözümlerin sürdürülebilirliğini inceleyebilme firsatı sunmuştur. Ayrıca çalışmada elde edilen sonuç, konu ile ilgili literatürdeki üçüncü mertebeden lineer olmayan diferansiyel denklemlerin çözümlerinin niteliksel davranışları üzerine var olan sonuçları geliştirecek ve bu kapsamda çalışma yapan araştırmacılara yardımcı olacak niteliktedir.

$\varphi_{0}, \varphi_{1}, a_{0}, a_{1}, n, N, \delta_{i}, \mu_{i}, \sigma_{i}, g_{1}, f_{0}$ ve $f_{1}$ pozitif sabitler olmak üzere her $t \geq 0$ için aşağıdaki şartlar yazılabilir.

A1) $0<\varphi_{0} \leq \varphi(x) \leq \varphi_{1}, \int_{-\infty}^{\infty}\left|\varphi^{\prime}(u)\right| d u<\infty$,

A2) $a_{0} \leq a(t) \leq a_{1}, a^{\prime}(t) \leq 0$,

A3) $0<n \leq c(t) \leq b(t) \leq N, b^{\prime}(t) \leq c^{\prime}(t) \leq 0$,

A4) $h_{i}(0)=0, \frac{h_{i}(x)}{x} \geq \delta_{i},(x \neq 0),\left|h_{i}{ }^{\prime}(x)\right| \leq \mu_{i}$,

A5) $g(x, 0)=0, \varphi_{1} \sum_{i=1}^{n} \sigma_{i} \leq \frac{g(x, y)}{y} \leq g_{1},(y \neq 0) \quad g_{x}(x, y) \leq 0$,

A6) $f_{0} \leq f(t, x, y) \leq f_{1}, f_{t}(t, x, y) \leq 0, \quad y f_{x}(t, x, y) \leq 0$,

A7) $\int_{0}^{\infty}|p(t)| d t<\infty$.

\section{Temel sonuç}

Temel sonucumuzun gösteriminde ihtiyacımız olan ve iyi bilinen

$\frac{d x}{d t}=F(t, x)$ 
otonom olmayan diferansiyel denklem sistemini ele alalım. Burada $x, n$ boyutlu bir vektör, $t \in[0, \infty)$ ve $D, \mathbb{R} \times \mathbb{R}^{n}$ kümesinde açık bir küme olmak üzere $F(t, x), D$ kümesi üzerindeki bir $(t, x)$ noktasında sürekli bir fonksiyondur.

Teorem 1. $F \in C(D)$ ve $D$ üzerinde $|F| \leq M$ olsun. $\varphi, j=(\alpha, \beta)$ aralığ üzerinde (4) denklem sisteminin bir çözümü olmak üzere eğer,

i) $\lim _{t \rightarrow \alpha^{+}} \varphi(t)=\varphi\left(\alpha^{+}\right)$ve $\lim _{t \rightarrow \beta^{-}} \varphi(t)=\varphi\left(\beta^{-}\right)$vardir.

ii) $\left(\alpha, \varphi\left(\alpha^{+}\right)\right)$, (ayrıca $\left.\left(\beta, \varphi\left(\beta^{-}\right)\right)\right) D$ kümesinin içindedir.

Şartları sağlıyorsa sırasıyla $\varphi, t=\alpha$ noktasının soluna veya $t=\beta$ noktasının sağına sürdürülebilirdir.

İspat. Hsu [22].

Teorem 2. (A1)-(A7) şartlarının sağladığını varsayalım. Buna göre eğer,

$\frac{N \varphi_{1}}{n \varphi_{0}} \sum_{i=1}^{n} \sigma_{i}^{-1} \mu_{i}<\alpha<\frac{a_{0} \varphi_{0} f_{0}}{\varphi_{1}}$

ve

$\xi<\min \left\{\frac{\frac{a n}{\varphi_{1}} \sum_{i=1}^{n} \sigma_{i}-\frac{N}{\varphi_{0}} \sum_{i=1}^{n} \mu_{i}}{\sum_{i=1}^{n} \lambda_{i}+\frac{a N}{2 \varphi_{0}^{2}} \sum_{i=1}^{n} \mu_{i}}, \frac{\frac{a_{0} f_{0}}{\varphi_{1}}-\frac{\alpha}{\varphi_{0}}}{\frac{N}{2 \varphi_{0}} \sum_{i=1}^{n} \mu_{i}}\right\}$

ise (2) denkleminin tüm çözümleri sınırlı ve sürdürülebilirdir.

İspat.

$V(t)=e^{-\frac{\rho(t)}{\varepsilon}} U(t, x(t), y(t), z(t))$

biçimindeki $V(t)=V(t, x(t), y(t), z(t))$ sürekli türevlenebilir Lyapunov fonksiyonunu tanımlayalım. Burada $\varepsilon$ pozitif bir sabit olup daha sonra tanımlanacaktır,

$\Omega(t)=\frac{x^{\prime}(t) \varphi^{\prime}(x(t))}{\varphi^{2}(x(t))}, \alpha_{1}(t)=\min \{x(0), x(t)\}, \alpha_{2}(t)=\max \{x(0), x(t)\}$ olmak üzere

$\rho(t)=\int_{0}^{t}|\Omega(s)| d s=\int_{0}^{t}\left|\frac{x^{\prime}(s) \varphi^{\prime}(x(s))}{\varphi^{2}(x(s))}\right| d s=\int_{\alpha_{1}(t)}^{\alpha_{1}(t)}\left|\frac{\varphi^{\prime}(u)}{\varphi^{2}(u)}\right| d u$

$\leq \frac{1}{\varphi_{0}^{2}} \int_{-\infty}^{+\infty}\left|\varphi^{\prime}(u)\right| d u<\infty$

ve $\lambda_{\mathrm{i}}$ daha sonra tanımlanacak olan pozitif sabitler olup

$U(t)=U(t, x(t), y(t), z(t))=\alpha c(t) \sum_{i=1}^{n} \int_{0}^{x} h_{i}(u) d u+c(t) y \sum_{i=1}^{n} h_{i}(x)$
$+b(t) \varphi(x) \int_{0}^{\frac{y}{\varphi(x)}} g(x, \tau) d \tau+\frac{1}{2} z^{2}+\frac{\alpha}{\varphi(x)} y z+\alpha a(t) \int_{0}^{\frac{y}{\varphi(x)}} f(t, x, \tau) \tau d \tau$ 
$+\sum_{\mathrm{i}=1}^{\mathrm{n}} \lambda_{\mathrm{i}} \int_{-\eta_{i}(t)}^{0} \int_{t+s}^{t} y^{2}(u) d u d s$

dir. $V(t)$ Lyapunov fonksiyonunun pozitif tanımlı olduğunu gösterelim.

$\sum_{\mathrm{i}=1}^{\mathrm{n}} \lambda_{\mathrm{i}} \int_{-\eta_{i}(t)}^{0} \int_{t+s}^{t} y^{2}(u) d u d s$

ifadesi negatif olmayan bir integral olduğundan (6) denkleminden

$U(t) \geq c(t)\left(\alpha \sum_{i=1}^{n} \int_{0}^{x} h_{i}(u) d u+y \sum_{i=1}^{n} h_{i}(x)+\frac{b(t)}{c(t)} \varphi(x) \int_{0}^{\frac{y}{\varphi(x(s))}} g(x, \tau) d \tau\right)$

\section{$\frac{y}{\varphi(x)}$}

$+\frac{1}{2} z^{2}+\frac{\alpha}{\varphi(x)} y z+\alpha a(t) \int_{0} f(t, x, \tau) \tau d \tau$

yazılabilir. Buradan (A1)-(A6) şartları göz önüne alındığında,

$U(t) \geq c(t)\left(\begin{array}{c}\sum_{i=1}^{n} \int_{0}^{x}\left(\alpha-\sigma_{i}{ }^{-1} h_{i}{ }^{\prime}(u)\right) h_{i}(u) d u+ \\ +\frac{1}{2} \sum_{i=1}^{n} \sigma_{i}{ }^{-1}\left(h_{i}(x)+\sigma_{i} y\right)^{2} \\ +\varphi(x) \int_{0}^{\frac{y}{\varphi(x(s))}}\left(\frac{g(x, \tau)}{\tau}-\varphi(x) \sum_{i=1}^{n} \sigma_{i}\right) \tau d \tau\end{array}\right)$

$+\frac{1}{2}\left(z+\frac{\alpha}{\varphi(x)} y\right)^{2}+\alpha a(t) \int_{0}\left(f(t, x, \tau)-\frac{\alpha}{a(t)}\right) \tau d \tau$

$\geq\left(\sum_{i=1}^{n} \int_{0}^{x}\left(\alpha-\sigma_{i}^{-1}\right) \frac{h_{i}(u)}{u} u d u\right)+\frac{1}{2}\left(z+\frac{\alpha}{\varphi(x)} y\right)^{2}+\alpha a_{0} \int_{0}^{\frac{y}{\varphi(x)}}\left(f_{0}-\frac{\alpha}{a_{0}}\right) \tau d \tau$

$\geq \frac{1}{2}\left(\sum_{i=1}^{n} n\left(\alpha-\sigma_{i}^{-1} \mu_{i}\right) \delta_{i}\right) x^{2}+\frac{1}{2}\left(z+\frac{\alpha}{\varphi(x)} y\right)^{2}+\alpha \frac{a_{0} f_{0}-\alpha}{2 \varphi_{1}^{2}} y^{2}$

olur. Böylece

$\frac{N \varphi_{1}}{n \varphi_{0}} \sum_{i=1}^{n} \sigma_{i}^{-1} \mu_{i}<\alpha<\frac{a_{0} \varphi_{0} f_{0}}{\varphi_{1}}$

olduğundan $K_{1}$ pozitif bir sabit olmak üzere

$U(t) \geq K_{1}\left(x^{2}+y^{2}+z^{2}\right)$ 
elde edilir. Bu eşitsizlik $V(t) \geq 0$ olmasını gerektirir. Bu ise (5) de tanımlanan $V(t)$ Lyapunov fonksiyonunun pozitif tanımlı olduğu anlamına gelir.

Şimdi ise $V(t)$ Lyapunov fonksiyonunun sınırlı olduğunu gösterelim. (2) denkleminin herhangi bir $(x(t), y(t), z(t))$ çözümünü alalım. Buna göre $U(t)$ fonksiyonunun $t$ değişkenine göre türevi alındığında,

$$
\begin{aligned}
& U^{\prime}=\alpha c^{\prime}(t) \sum_{i=1}^{n} \int_{0}^{x} h_{i}(u) d u+c^{\prime}(t) y \sum_{i=1}^{n} h_{i}(x)+\frac{c(t)}{\varphi(x)} y^{2} \sum_{i=1}^{n} h_{i}{ }^{\prime}(x) \\
& +b^{\prime}(t) \varphi(x) \int_{0}^{\frac{y}{\varphi(x)}} g(x, \tau) d \tau+b(t) \Omega(t) \varphi^{2}(x) \int_{0}^{\frac{y}{\varphi(x)}} g(x, \tau) d \tau+b(t) y \int_{0} g_{x}(x, \tau) d \tau \\
& -b(t) \Omega(t) \varphi(x) g\left(x, \frac{y}{\varphi(x)}\right) y+p(t) z-\frac{a(t)}{\varphi(x)} f\left(t, x, \frac{y}{\varphi(x)}\right) z^{2} \\
& -\frac{a(t) \varphi^{\prime}(x)}{\varphi^{3}(x)} f\left(t, x, \frac{y}{\varphi(x)}\right) z y^{2}+c(t) z \sum_{i=1}^{n} \int_{t-\tau_{i}}^{t} \frac{y(s)}{\varphi(x(s))} h_{i}{ }^{\prime}(x(s)) d s \\
& -\alpha \Omega(t) y z+\frac{\alpha}{\varphi(x)} z^{2}+\frac{\alpha}{\varphi(x)} p(t) y-\frac{\alpha b(t)}{\varphi(x)} g\left(x, \frac{y}{\varphi(x)}\right) y \\
& +\frac{\alpha c(t) y \sum_{i=1}^{n}}{\varphi(x)} \sum_{t-\tau_{i}}^{t} \frac{y(s)}{\varphi(x(s))} h_{i}{ }^{\prime}(x(s)) d s+\alpha a^{\prime}(t) \int_{0}^{y(x)} f(t, x, \tau) \tau d \tau \\
& +\alpha a(t) \int_{0}^{n} f_{t}(t, x, \tau) \tau d \tau+\frac{\alpha a(t) y}{\varphi(x)} \int_{0}^{t} f_{x}(t, x, \tau) \tau d \tau+\sum_{i=1}^{n} \lambda_{i} \eta_{i}(t) y^{2} \\
& -\sum_{i=1}^{n} \lambda_{\mathrm{i}}\left(1-\eta_{i}{ }^{\prime}(t)\right) \\
& \int^{2}(s) d s \\
& t-\eta_{i}(t)
\end{aligned}
$$

elde edilir. Bu eşitlik düzenlenirse

$$
\begin{aligned}
& U^{\prime}=c^{\prime}(t)\left(\alpha \sum_{i=1}^{n} \int_{0}^{x} h_{i}(u) d u+y \sum_{i=1}^{n} h_{i}(x)+\frac{b^{\prime}(t)}{c^{\prime}(t)} \varphi(x) \int_{0}^{\frac{y}{\varphi(x)}} g(x, \tau) d \tau\right) \\
& +\left(\frac{c(t)}{\varphi(x)} \sum_{i=1}^{n} h_{i}^{\prime}(x)-\frac{\alpha b(t)}{\varphi(x)} \frac{g\left(x, \frac{y}{\varphi(x)}\right)}{y}+\sum_{i=1}^{n} \lambda_{\mathrm{i}} \eta_{i}(t)\right) y^{2} \\
& +\frac{1}{\varphi(x)}\left(\alpha-a(t) f\left(t, x, \frac{y}{\varphi(x)}\right)\right) z^{2}+b(t) y \int_{0}^{\frac{y}{\varphi(x)}} g_{x}(x, \tau) d \tau
\end{aligned}
$$




$$
\begin{aligned}
& +\alpha a^{\prime}(t) \int_{0}^{\frac{y}{\varphi(x)}} f(t, x, \tau) \tau d \tau+\alpha a(t) \int_{0}^{\frac{y}{\varphi(x)}} f_{t}(t, x, \tau) \tau d \tau+\frac{\alpha a(t) y}{\varphi(x)} \int_{0}^{\frac{y}{\varphi(x)}} f_{x}(t, x, \tau) \tau d \tau \\
& +c(t) z \sum_{i=1}^{n} \int_{t-\tau_{i}}^{t} \frac{y(s)}{\varphi(x(s))} h_{i}{ }^{\prime}(x(s)) d s+\frac{\alpha c(t) y}{\varphi(x)} \sum_{i=1}^{n} \int_{t-\tau_{i}}^{t} \frac{y(s)}{\varphi(x(s))} h_{i}{ }^{\prime}(x(s)) d s \\
& -\sum_{\mathrm{i}=1}^{\mathrm{n}} \lambda_{\mathrm{i}}\left(1-\eta_{i}{ }^{\prime}(t)\right) \int_{t-\eta_{i}(t)} y^{2}(s) d s+\Omega(t)\left(\begin{array}{l}
\frac{y}{\varphi(x)} \\
-b(t) \varphi^{2}(x) \int_{0}^{t} g(x, \tau) d \tau \\
-
\end{array}\right) \\
& +\Omega(t)\left(a(t) f\left(t, x, \frac{y}{\varphi(x)}\right)-\alpha\right) z y+p(t)\left(\frac{\alpha}{\varphi(x)} y+z\right)
\end{aligned}
$$

elde edilir. Böylece, (A1)-(A7) şartları ve $|u v| \leq \frac{1}{2}\left(u^{2}+v^{2}\right)$ eşitsizliği göz önüne alınırsa son eşitlikten,

$$
\begin{aligned}
& U^{\prime}(t) \leq c^{\prime}(t)\left(\begin{array}{c}
\sum_{i=1}^{n} \int_{0}^{x}\left(\alpha-\sigma_{i}{ }^{-1} h_{i}{ }^{\prime}(u)\right) h_{i}(u) d u \\
+\frac{1}{2} \sum_{i=1}^{n} \sigma_{i}{ }^{-1}\left(h_{i}(x)+\sigma_{i} y\right)^{2} \\
+\varphi(x) \int_{0}^{\frac{y}{\varphi(x)}}\left(\frac{g(x, \tau)}{\tau}-\varphi(x) \sum_{i=1}^{n} \sigma_{i}\right) \tau d \tau
\end{array}\right) \\
& +\left(\frac{\alpha}{\varphi_{0}}-\frac{a_{0} f_{0}}{\varphi_{1}}+\frac{N \xi}{2 \varphi_{0}} \sum_{i=1}^{n} \mu_{i}\right) z^{2}+p(t)\left(\frac{\alpha}{\varphi_{0}} y^{2}+z^{2}+\frac{\alpha}{\varphi_{0}}+1\right) \\
& +\left(\begin{array}{c}
\frac{N}{\varphi_{0}} \sum_{i=1}^{n} \mu_{i}-\frac{\alpha n}{\varphi_{1}} \sum_{i=1}^{n} \sigma_{i} \\
+\xi\left(\sum_{i=1}^{n} \lambda_{i}+\frac{\alpha N}{2 \varphi_{0}^{2}} \sum_{i=1}^{n} \mu_{i}\right)
\end{array}\right) y^{2}+\left(\begin{array}{c}
\left(\frac{N}{2 \varphi_{0}}+\frac{\alpha N}{\varphi_{0}{ }^{2}}\right) \sum_{i=1}^{n} \mu_{i} \\
-\sum_{\mathrm{i}=1}^{n} \lambda_{\mathrm{i}}(1-\rho)
\end{array}\right) \int_{t-\eta_{i}(t)}^{t} y^{2}(s) d s \\
& +|\Omega(t)|\left(\begin{array}{c}
\left(N g_{1}+\frac{a_{1} f_{1}-\alpha}{2}-\alpha\right) y^{2} \\
+\left(\frac{a_{1} f_{1}-\alpha}{2}\right) z^{2}
\end{array}\right)
\end{aligned}
$$

ifadesi elde edilir. Burada

$$
\sum_{\mathrm{i}=1}^{\mathrm{n}} \lambda_{\mathrm{i}}(1-\rho)=\left(\frac{N}{2 \varphi_{0}}+\frac{\alpha N}{\varphi_{0}{ }^{2}}\right) \sum_{i=1}^{n} \mu_{i}
$$


olmak üzere

$\frac{N \varphi_{1}}{n \varphi_{0}} \sum_{i=1}^{n} \sigma_{i}^{-1} \mu_{i}<\alpha<\frac{a_{0} \varphi_{0} f_{0}}{\varphi_{1}}$

ve

$\xi<\min \left\{\frac{\frac{\alpha n}{\varphi_{1}} \sum_{i=1}^{n} \sigma_{i}-\frac{N}{\varphi_{0}} \sum_{i=1}^{n} \mu_{i}}{\sum_{i=1}^{n} \lambda_{i}+\frac{\alpha N}{2 \varphi_{0}^{2}} \sum_{i=1}^{n} \mu_{i}}, \frac{\frac{a_{0} f_{0}}{\varphi_{1}}-\frac{\alpha}{\varphi_{0}}}{\frac{N}{2 \varphi_{0}} \sum_{i=1}^{n} \mu_{i}}\right\}$

olduğundan

$U^{\prime}(t) \leq|\Omega(t)| K_{2}\left(x^{2}+y^{2}\right)+p(t) K_{3}\left(y^{2}+z^{2}+1\right)$

olur. Burada $K_{2}=g_{1}+\frac{a_{1} f_{1}-\alpha}{2}$ ve $K_{3}=\max \left\{1, \frac{\alpha}{\varphi_{0}}\right\}$ dir. Böylece (5) ile tanımlanan $V(t)$ fonksiyonunun (3) sisteminin çözümleri boyunca türevi alınırsa

$V^{\prime}(t)=e^{-\frac{\rho(t)}{\varepsilon}}\left(-\frac{|\Omega(t)|}{\varepsilon} U(t)+U^{\prime}(t)\right)$

elde edilir. Burada $\varepsilon=\frac{K_{1}}{K_{2}}$ olmak üzere $e^{-\frac{\rho(t)}{\varepsilon}} \leq 1$ olduğundan (7) ve (8) kullanılarak

$V^{\prime}(t)=e^{-\frac{\rho(t)}{\varepsilon}}\left(\begin{array}{c}-|\Omega(t)| K_{2}\left(y^{2}+z^{2}\right) \\ +|\Omega(t)| K_{2}\left(y^{2}+z^{2}\right) \\ +p(t) K_{3}\left(y^{2}+z^{2}+2\right)\end{array}\right) \leq e^{-\frac{\rho(t)}{\varepsilon}} p(t) K_{3}\left(y^{2}+z^{2}+2\right)$

$\leq 2 K_{3} p(t)+K_{4} p(t) V(t)$

yazılabilir. Burada $K_{4}=\frac{K_{3}}{K_{1}}$ olup son eşitsizliğin her iki tarafının 0 dan $t(t \geq 0)$ ye integrali alınırsa

$V(t) \leq V(0)+2 K_{3} \int_{0}^{t}|p(s)| d s+K_{3} \int_{0}^{t}|p(s)||V(s)| d s \leq K_{5}+K_{3} \int_{0}^{t}|p(s)||V(s)| d s$

olur. Burada

$K_{5}=V(0)+2 K_{3} \int_{0}^{t}|p(s)| d s$

dir. Böylece son eşitsizlikte Gronwall-Reid-Bellman eşitsizliği kullanılarak

$V(t) \leq K_{5} \exp \left(K_{4} \int_{0}^{t}|p(s)| d s\right)=K_{6}$

elde edilir. Bu ise $V(t)$ Lyapunov fonksiyonunun sınırlı olduğu anlamına gelir. 
(3) sisteminin, Cauchy-Peano varlık teoreminden dolayı, $a>0$ olmak üzere $\left[t_{0}, t_{0}+a\right.$ ) aralığındaki bir çözümü $(x(t), y(t), z(t))$ olsun. $\mathrm{Bu}$ aralığın $\left[t_{0}, \infty\right)$ aralığına genişletilebileceğini göstermek istiyoruz. O halde bunun tersini kabul edelim. Yani $T<$ $\infty$ olmak üzere (3) sisteminin $\left[t_{0}, T\right)$ aralığında bir çözümü var ve

$\lim _{t \rightarrow T^{-}}(|x(t)|+|y(t)|+|z(t)|)=\infty$

olduğunu varsayalım. (3) sisteminin $\left(x_{0}, y_{0}, z_{0}\right)$ başlangıç şartları altındaki bir $(x(t), y(t), z(t))$ çözümünü ele alalım. Böylece (5),(7) ve (9) dan

$\left|x^{2}(T)\right|+\left|y^{2}(T)\right|+\left|z^{2}(T)\right| \leq \frac{K_{6}}{K_{1}} e^{\frac{\rho(T)}{\varepsilon}}=K_{7}$,

yazılabilir. Buradan $t \rightarrow T^{-}$için $|x(t)|,|y(t)|$ ve $|z(t)|$ nin sinırlı olduğu kolayca görülür. $\mathrm{Bu}$ ise varsayımımızla çelişir ve $T<\infty$ olmasının mümkün olmadığını $T=\infty$ olması gerektiğini gösterir. Böylece ispat tamamlanır.

Örnek. Üçüncü mertebeden lineer olmayan $\eta_{1}(t)>0$ ve $\eta_{2}(t)>0$ iki değişken gecikmeli

$$
\begin{aligned}
& \left(\left(1+\frac{1}{1+x^{2}}\right) x^{\prime \prime}\right)^{\prime}+\left(4+\frac{1}{1+t}\right)\left(4+\frac{1}{1+y^{2}}\right) x^{\prime \prime}+\left(2+\frac{1}{1+t}\right)\left(12 y+\frac{y}{1+y^{2}}\right) \\
& +2\left(2+\frac{1}{2+t}\right) x\left(t-\eta_{1}(t)\right)+2\left(2+\frac{1}{2+t}\right) x\left(t-\eta_{2}(t)\right)=\frac{1}{1+t^{2}}
\end{aligned}
$$

diferansiyel denklemini ele alalım. (10) denklemi (2) denklemi ile karşılaştırıldığında

$$
\begin{aligned}
& \varphi_{0} \leq \varphi(x)=1+\frac{1}{1+x^{2}} \leq 2=\varphi_{1}, \int_{-\infty}^{+\infty}\left|\varphi^{\prime}(u)\right| d u<\int_{-\infty}^{+\infty}\left|\frac{2 u}{\left(1+u^{2}\right)^{2}}\right| d u=0<\infty, \\
& a_{0}=4 \leq a(t)=4+\frac{1}{1+t} \leq 5=a_{1}, a^{\prime}(t)=-\frac{1}{(1+t)^{2}} \leq 0 \\
& b(t)=2+\frac{1}{1+t}, b^{\prime}(t)=-\frac{1}{(1+t)^{2}}, c(t)=2+\frac{1}{2+t}, c^{\prime}(t)=-\frac{1}{(2+t)^{2}} \\
& n=2 \leq c(t) \leq b(t) \leq 3=N, b^{\prime}(t) \leq c^{\prime}(t) \leq 0 \\
& f_{0}=4 \leq f\left(t, x, x^{\prime}\right)=4+\frac{1}{1+y^{2}} \leq 5=f_{1}, f_{t}\left(t, x, x^{\prime}\right)=0, y f_{x}\left(t, x, x^{\prime}\right)=0, \\
& g(x, 0)=0 \text { and } y \neq 0, g_{0}=12 \leq \frac{g(x, y)}{y}=12+\frac{1}{1+y^{2}} \leq 13=g_{1}, \\
& h_{i}(0)=0, \frac{h_{i}(x)}{x}=2=\delta_{i},\left|h_{i}{ }^{\prime}(x)\right|=2=\mu_{i}, \\
& (i=1,2)
\end{aligned}
$$

Ve

$$
\int_{0}^{\infty} p(s) d s=\int_{0}^{\infty} \frac{d s}{1+s^{2}}=\frac{\pi}{2}<\infty .
$$


ifadeleri yazılabilir. Sonuç olarak $\sigma_{1}=\sigma_{2}=3$ olmak üzere (10) denklemi için (A1)(A7) şartları sağladığından bu denklemin tüm çözümleri sınırlı ve sürdürülebilirdir.

Ayrıca (10) denkleminin çözümlerinin yörüngeleri aşağıdaki şekil 1 de gösterilmiştir.

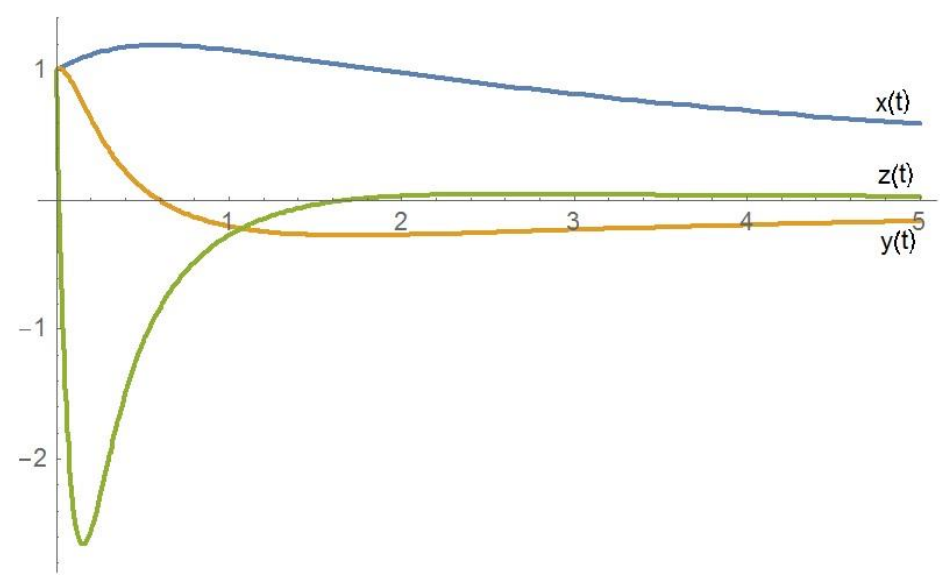

Şekil 1. $x(t), y(t)$ ve $z(t)$ çözümlerinin zamana göre değişimi.

\section{Sonuç ve tartışma}

$\mathrm{Bu}$ çalışmada daha önce ele alınmamış lineer olmayan değişken gecikmeli bir diferansiyel denklem göz önüne alındı ve Lyapunov'un ikinci metodu kullanılarak bu denklemin çözümlerinin sinırlılığı ve sürdürülebilirliği üzerine yeni bir sonuç elde edildi. Elde edilen sonuç ele alınan denklemin çözümlerinin sınırlı bir aralıkta değil de sonsuz bir aralıkta davranışlarının incelenmesine olanak sağlayacak niteliktedir. Bu yönüyle çalışmamız bu alanda bir ilk olması sebebiyle çalışma yapacak olan araştırmacılara farklı denklemler üzerinde yöntemi kullanmaları adına ilham kaynağı olup daha kararlı sonuçlar elde etmelerine olanak sağlayacaktır.

\section{Kaynaklar}

[1] Liapunov, A. M., Stability of Motion.With a Contribution by V.A. Pliss and an introduction by V.P. Basov, Mathematics in Science and Engineering, vol. 30. Academic Press, New York-London. Translated from the Russian by Flavian Abramovici and Michael Shimshoni, ( 1966).

[2] Ahmad, S. and Rama Mohana Rao, M., Theory of ordinary differential equations with applications in biology and engineering affiliated. East-West Press Pvt. Ltd., New Delhi, (1999).

[3] Burton, T. A., Stability and periodic solutions of ordinary and functional differential equations, Mathematics in Science and Engineering, Academic Press, Orlando, Fla, USA, (1985).

[4] Driver, R. D., Ordinary and delay differential equations. Springer, NewYork, NY, USA, (1976).

[5] Hale, J. K., Theory of functional diflerential equations. Springer-Verlag, New York, (1977).

[6] Krasovskii, N. N., Stability of motion. Applications of Lyapunov's second method to differential systems and equations with delay, Translated by J. L. Brenner Stanford University Press, Stanford, California, (1963). 
[7] Reissig, R., Sansone, G. and Conti, R., Non-linear differential equations of higher order, Noordhoff International Publishing, (1974).

[8] Yoshizawa, T., 1966. Stability Theory by Lyapunov's Second Method, Mathematical Society of Japan, No. 9, Tokyo.

[9] Abou-El-Ela, A. M. A., Sadek, A. I. and Mahmoud, A. M., Stability and boundedness of solutions of certain third order non linear delay differential equation, ICGST-ACSE Journal, 9, 1, 9-15, (2009).

[10] Ademola, A. T., Ogundare, B. S., Ogundiran, M. O. and Adesina, O. A., Stability, boundedness, and existence of periodic solutions to certain third-order delay differential equations with multiple deviating Arguments, International Journal of Differential Equations, Art. ID 213935, 12 pp, (2015).

[11] Ademola, A. T., Existence and uniqueness of a periodic solution to certain third order nonlinear delay differential equation with multiple deviating arguments, Acta Universitatis Sapientiae,Mathematica, 5, 2, 113-131,(2013).

[12] Ayhan, T., (2017). A note on the continuability and boundedness of solutions to a class of vector differential equations of third order with finite delay, Quaestiones Mathematicae, 40, 8, 1095-1109, (2017).

[13] Ayhan, T. and Sofuoglu, Y., Global existence and boundedness of a certain nonlinear vector integro-differential equation of second order with multiple deviating arguments, Mathematıcal Communıcatıons, 22, 165-176, (2017).

[14] Remili, M. and Oudjedi, L. D., Stability and boundedness of the solutions of nonautonomous third order differential equations with delay, Acta Univ. Palack.Olomuc. Fac. Rerum.Natur. Math., 53, 2, 139-147, (2014).

[15] Remili, M. and Oudjedi, L. D., Uniform ultimate boundedness and asymptotic behaviour of third order nonlinear delay differential equation, Afrika Matematika, 27, 7, 1227-1237, (2016).

[16] Remili, M. and Beldjerd, D., A boundedness and stability results for a kind of third order delay differential equations, Applications \& Applied Mathematics, 10, 2, 772-782, (2015).

[17] Tunç, C. and Ayhan, T., Continuability and boundedness of solutions for a kind of nonlinear delay integro differential equations of the third order, Journal of Mathematical Sciences, 236, 3, 354-366, (2019).

[18] Tunç, C. and Ayhan, T., Global existence and boundedness of solutions of a certain nonlinear integro-differential equation of second order with multiple deviating arguments, Journal of Inequalities and Applications, 46, (2016).

[19] Tunç, C. and Ayhan, T., On the global existence and boundedness of solutions to a nonlinear integro-differential equations of second order, Journal of Interpolation and Approximation in Scientific Computing, 2, 84-97, (2015).

[20] Tunç, C. and Ayhan, T., New boundedness results for a kind of nonlinear differential equations of third order, Journal of Computational Analysis and Applications, 13, 3, 477-484, (2011).

[21] Tunç, C., New results about stability and boundedness of solutions of certain non-linear third order delay differential equations. The Arabian Journal for Science and Engineering, 31, 2A, 185-196, (2006).

[22] Hsu, S. B., Ordinary differential equations with applications, World Scientific Publishing Co. Pte. Ltd., 244, (2006). 